\title{
A Novel Seepage Safety Monitoring Model of CFRD with Slab Cracks Using Monitoring Data
}

\author{
Zhongwen Shi, ${ }^{1,2,3}$ Chongshi Gu $\mathbb{D}^{1,2,3}$ Erfeng Zhao $\mathbb{D}^{1,2,3}$ and Bo Xu $\mathbb{D}^{4}$ \\ ${ }^{1}$ College of Water Conservancy and Hydropower Engineering, Hohai University, Nanjing 21009, China \\ ${ }^{2}$ State Key Laboratory of Hydrology-Water Resources and Hydraulic Engineering, Hohai University, Nanjing 210098, China \\ ${ }^{3}$ National Engineering Research Center of Water Resources Efficient Utilization and Engineering Safety, Hohai University, \\ Nanjing 210098, China \\ ${ }^{4}$ College of Hydraulic Science and Engineering, Yangzhou University, Yangzhou 225009, China
}

Correspondence should be addressed to Chongshi Gu; csgu@hhu.edu.cn

Received 13 August 2019; Revised 4 February 2020; Accepted 12 February 2020; Published 5 May 2020

Academic Editor: Qiuye Sun

Copyright (C) 2020 Zhongwen Shi et al. This is an open access article distributed under the Creative Commons Attribution License, which permits unrestricted use, distribution, and reproduction in any medium, provided the original work is properly cited.

The traditional regression model usually simulates the influence of water pressure and rainfall in the early stage based on experience, but it is not suitable. To solve this problem, the normal distribution curve is used to simulate the lagging effect of water pressure and rainfall on dam seepage. In view of problem of slab cracks, the influence of cracks on seepage is analyzed. In this paper, a safety monitoring model for concrete face rockfill dam (CFRD) seepage with cracks considering the lagging effect is proposed, in which slab cracks are considered as an influencing factor. The radial basis function neural network (RBFNN) optimized by genetic algorithm (GA) is used to establish a safety monitoring model for a CFRD seepage. Seepage of the dam is predicted by this model, whose results are similar to the monitoring data, which indicates that the method has certain applicability. Through the analysis of the proportion of factors affecting CFRD seepage, it is found that the rainfall component has the greatest impact on the total seepage, accounting for more than $50 \%$, and the crack component accounts for about $10 \%$. Finally, through the cloud model, the monitoring index of CFRD seepage is worked out, which has certain guiding significance for the treatment of abnormal seepage monitoring data.

\section{Introduction}

Seepage has been a major concern in dam engineering since the face rockfill dam emerged. The expansion of concrete face slab cracks and the deterioration of joint water stop are liable to lead to seepage failure of cushion, transition layer, and rockfill body of CFRD, which has serious negative impact on dam deformation, structure, and safety [1-3]. The deterioration of impervious state of concrete face slab and cushion will increase the monitoring data of weir behind the dam. It is important to establish a safety monitoring model of seepage for CFRD considering cracks in the face slab and draw up the monitoring index of seepage for timely grasping the operation state of CFRD and ensuring the safety of the dam [4-9].

The influencing factors of seepage of CFRD are complex, including water level, rainfall, aging, and temperature. In the research of the safety monitoring model of seepage, experts and scholars have carried out much research, including statistical model, deterministic model, and mixed model [10]. Shen and $\mathrm{Wu}[11]$ established the monitoring model of uplift pressure of dam foundation by stepwise regression analysis under different combinations of time-effect factors and water level factors. Cen [12] studied the seepage field characteristics of each part and the whole of CFRD. According to the dam engineering theory, the mathematical expressions of reservoir water level, rainfall, temperature, and time components were deduced. The statistical model of seepage monitoring for CFRD was established, and a high precision was achieved in the seepage safety monitoring of Nalan CFRD. However, the monitoring data of dam seepage lag behind the changes in water level and rainfall in the upstream reservoir, so the lagging effect has to be taken into account in the construction of seepage safety monitoring model. 
Zhang et al. [13] introduced the lagging effect function of reservoir water level and rainfall, determined the lagging days and the impact days, and then established the seepage lagging monitoring model. Cai [14] studied the seepage statistical model considering lagging effect based on the influence of the change rate of reservoir water level on dam seepage. Chen et al. [15] established a coupled model of unsteady seepage and nonlinear displacement (modeling coupled processes of nonsteady seepage flow and nonlinear deformation for a CFRD). Xu et al. [16] applied the cloud adaptive genetic algorithm in the field of constructing the seepage safety monitoring model of CFRD considering the influence of upstream water level and rainfall on seepage. The fitting accuracy and prediction effect of the model are better than the traditional seepage statistical model. Chen et al. [17] proposed a spatiotemporal clustering and diagnosis method for concrete arch dams based on deformation monitoring data. In recent years, artificial neural network, fuzzy mathematics, genetic algorithm, and grey system have been applied to dam safety monitoring field [18-22]. Tian et al. [23] established a seepage safety monitoring model of earth-rock dam by combining the monitoring data based on the principle of artificial neural network; Zhang [24] used the optimization ability of particle swarm and artificial bee colony algorithm to establish the corresponding support vector machine seepage monitoring model. Stojanovic et al. [25] proposed a selftuning system for dam behavior modeling based on evolving artificial neural networks.

At present, the common monitoring index formulation methods include confidence interval method, typical small probability method, limit state method, simulation calculation method, mechanical calculation method, and structural analysis method, among which the first two methods are called the mathematical statistics method. In recent years, the maximum entropy method, cloud model method, Monte Carlo method, projection pursuit method, and so on have also appeared and achieved good results. $\mathrm{Wu}$ and $\mathrm{Wu}$ [26] simulated the nonlinear characteristics of dam body, strata, and bedrock, established a viscoelastic model of stressseepage coupling, and constructed a first-level monitoring index of deformation; in addition, they established an elasticplastic model of seepage-stress coupling and a second-level monitoring index. Guo et al. [27] established the viscoelastic finite element model of seepage and stress coupling and constructed the deformation monitoring index, which can accurately reflect the seepage and deformation characteristics of the dam after impoundment. Cong et al. [28] deduced the maximum entropy probability density function model based on information theory and applied it to the field of dam monitoring index formulation. Zhu et al. [29] applied cloud model theory to the formulation of dam deformation monitoring index and achieved good results.

In view of the shortcomings of the traditional statistical regression model of seepage [2,30-33], considering the lagging days of reservoir water level and rainfall, the lagging effects of water level and rainfall on seepage, and the cracking factors, the safety monitoring model of seepage of a CFRD is established, which is optimized by using GARBFNN. Based on the proposed model, the proportion of factors affecting CFRD seepage is worked out, and the influence of the cracks on the seepage of CFRD is found, accounting for about $10 \%$. Considering that the monitoring data of CFRD seepage is affected by many uncertainties, the seepage safety monitoring index is formulated based on the cloud model. The dam managers can take effective measures to reduce the influence of slab cracks on dam seepage to ensure dam safety according to the monitoring index.

\section{Materials and Methods}

\subsection{Traditional Seepage Safety Monitoring Model of CFRD}

2.1.1. Principle of Statistical Model. The seepage of CFRD is mainly affected by rainfall, reservoir water level, temperature, and aging. Therefore, the following statistical model is used in the analysis [10]:

$$
P=P_{H}+P_{T}+P_{U}+P_{\theta},
$$

where $P$ is the seepage of dam and $P_{H}, P_{T}, P_{U}$, and $P_{\theta}$ are the water pressure component, temperature component, rainfall component, and aging component, respectively.

\section{(1) Water pressure component $P_{H}$}

The change in upstream reservoir water level has a significant effect on dam seepage. The average values of 1-3 times of upstream water depth and average values of premonitoring water depth (1 day before the monitoring day, 23 days before the monitoring day, 4-7 days before the monitoring day, and 8-15 days before the monitoring day) are chosen as 7 factors in the model, namely,

$$
P_{H}=\sum_{i=1}^{3}\left[a_{i}\left(H_{u}^{i}-H_{u 0}^{i}\right)\right]+\sum_{i=4}^{7}\left[a_{i}\left(H_{u(i-3)}-H_{u 0}\right)\right]
$$

where $H_{u}$ is the upstream water level; $H_{u 0}$ is the initial monitoring data of upstream water level; $H_{u(i-3)}$ is the water level, average value of 1 day before monitoring day, average value of 2-3 days before monitoring day, average value of 4-7 days before monitoring day, and average value of 8-15 days before monitoring day $(i=4 \sim 7)$; and $a_{i}$ is the regression coefficient of water pressure component $r(i=4 \sim 7)$.

(2) Temperature component $P_{T}$

Factors of temperature components are selected as follows:

$$
\begin{aligned}
P_{T}= & \sum_{i=1}^{2}\left[b_{1 i}\left(\sin \frac{2 \pi i t}{365}-\sin \frac{2 \pi i t_{0}}{365}\right)\right. \\
& \left.+b_{2 i}\left(\cos \frac{2 \pi i t}{365}-\cos \frac{2 \pi i t_{0}}{365}\right)\right],
\end{aligned}
$$

where $t$ is the cumulative days from the initial monitoring day to the monitoring day, $t_{0}$ is the cumulative days from the initial monitoring day to the first monitoring day of the data sequence adopted in the model, and $b_{1 i}$ and $b_{2 i}$ are the regression coefficients of temperature factor.

(3) Rainfall component $P_{U}$ 
Rainfall has a certain effect on the change in dam seepage, which lags behind the rainfall for a certain time. Therefore, the mean values of precipitation in half a month before the dam seepage measurement can be selected as rainfall factors, that is,

$$
P_{U}=\sum_{i=1}^{5} c_{i}\left(U_{i}-U_{0 i}\right)
$$

where $U_{i}$ is the average rainfall on the monitoring day, average value of 1 day before monitoring day, average value of 2-3 days before monitoring day, average value of 4-7 days before monitoring day, and average value of 8-15 days before monitoring day $(i=1-5)$; $U_{0 i}$ is the average precipitation of each period in the initial monitoring day $(i=1-5)$; and $c_{i}$ is the regression coefficient of rainfall factor $(i=1-5)$.

\section{(4) Aging component $P_{\theta}$}

The composition of aging component is complex and closely related to dam body, lithology, fissures, structural distribution, and occurrence. The expression of aging component is selected as follows:

$$
P_{\theta}=d_{1}\left(\theta-\theta_{0}\right)+d_{2}\left(\ln \theta-\ln \theta_{0}\right),
$$

where $c_{1}$ and $c_{2}$ are the regression coefficients of aging component, $\theta$ is the cumulative day from the monitoring day to the initial monitoring day divided by 100 , and $\theta_{0}$ is the cumulative day from the initial monitoring day to the first monitoring day in the model divided by 100 .

In summary, the statistical model of CFRD seepage is as follows:

$$
\begin{aligned}
P= & P_{H}+P_{T}+P_{U}+P_{\theta} \\
= & a_{0}+\sum_{i=1}^{3}\left[a_{i}\left(H_{u}^{i}-H_{u 0}^{i}\right)\right]+\sum_{i=4}^{7}\left[a_{i}\left(H_{u(i-3)}-H_{u 0}\right)\right] \\
& +\sum_{i=1}^{2}\left[b_{1 i}\left(\sin \frac{2 \pi i t}{365}-\sin \frac{2 \pi i t_{0}}{365}\right)+b_{2 i}\left(\cos \frac{2 \pi i t}{365}-\cos \frac{2 \pi i t_{0}}{365}\right)\right] \\
& +\sum_{i=1}^{5} c_{i}\left(U_{i}-U_{0 i}\right)+\mathrm{d}_{1}\left(\theta-\theta_{0}\right)+\mathrm{d}_{2}\left(\ln \theta-\ln \theta_{0}\right)
\end{aligned}
$$

where $a_{0}$ is a constant, and the other symbolic meanings are identical equations (2)-(5).

\subsection{Seepage Safety Monitoring Model of CFRD with Cracks} considering Lagging Effect. The seepage of CFRD will be affected by the change in water level and temperature, as well as by rainfall and aging, among which the effect of reservoir water level and rainfall on seepage has lagging effect. The traditional statistical regression model fits and calculates on the premise of given preterm, but each dam has its own characteristics, and the lagging effect of water level and rainfall is different, so the traditional regression model often simulates the influence of prestage water level and rainfall based on experience does not have good applicability. In order to solve this problem, the normal distribution curve is adopted in this paper. The lagging effect of water level and rainfall on seepage flow is simulated by line, and the seepage safety monitoring model of CFRD is established. The lagging and influencing days of reservoir water level and rainfall on seepage of CFRD can be determined, which is helpful to analyze the evolution law of seepage control system of CFRD.

2.2.1. Water Pressure Component $P_{H}$. The lagging effect of reservoir water level on seepage is as follows:

$$
\begin{aligned}
Q(t)= & F\left[t, H(t), H\left(t-\tau_{1}(t)\right), H\left(t-\tau_{2}(t)\right), \ldots,\right. \\
& \left.H\left(t-\tau_{n}(t)\right)\right]
\end{aligned}
$$

where $\tau$ is the lag time between reservoir water level and monitoring seepage, $\tau_{k}(t) \geq 0, k=1,2, \ldots, n$, and $H(t)$ and $H\left(t-\tau_{n}(t)\right)$ are the reservoir water level.

Equation (7) reflects the lag relationship between reservoir water level and monitoring seepage. In this paper, an influence function is defined to express the lag effect caused by the change in reservoir water level.

Considering the influence of the change in water level on seepage $n$ days before monitoring day, the equivalent water level can be obtained as follows:

$$
H_{d}=\phi\left(H_{1}, H_{2}, \ldots, H_{k}, \zeta_{1}, \zeta_{2}, \ldots, \zeta_{k}\right),
$$

where $\zeta_{i}, i=1,2, \ldots, k(k \leq n)$, is the weight of the $i$ th water level, $\zeta=\left[\zeta_{1}, \zeta_{2}, \ldots, \zeta_{k}\right]$, and $\sum_{i=1}^{k} \zeta_{i}=1, k \leq n$, so equivalent water level $H_{d}$ can be obtained as follows:

$$
H_{d}=\sum_{i=1}^{k} \zeta_{i} H_{i}
$$

The influence of upstream reservoir water level on dam seepage is shown in Figure 1. The study shows that $\zeta(t)$ generally satisfied the normal distribution. If the lagging days and the influence days are expressed separately as $x_{1}$ and $x_{2}$, the lag effect function can be expressed as follows:

$$
\zeta(t)=\frac{1}{\sqrt{2 \pi} x_{2}} e^{-\left(t-x_{1}\right)^{2} / 2 x_{2}^{2}} .
$$

Suppose water storage began at time $t_{0}$, for a fixed time $t_{1}$,

$$
\int_{t_{0}}^{t_{1}} \zeta(t) d t=1
$$

In a certain period of time, the lag days $x_{1}$ and influence days $x_{1}$ of the same monitoring point are fixed values. $\zeta(t)$ in equation (11) is the lagging influence function of water level. Suppose the equivalent reservoir water level of seepage discharge at $t_{1}$ time is $H_{d}$, then

$$
H_{d}=\int_{t_{0}}^{t_{1}} \zeta(t) H(t) d t=\int_{t_{0}}^{t_{1}} \frac{1}{\sqrt{2 \pi} x_{2}} e^{-\left(t-x_{1}\right)^{2} / 2 x_{2}^{2}} H(t) d t,
$$

where $x_{1}$ is the day of water level lagging for seepage, $x_{2}$ is the standard deviation for seepage lagging, and $H(t)$ is the 


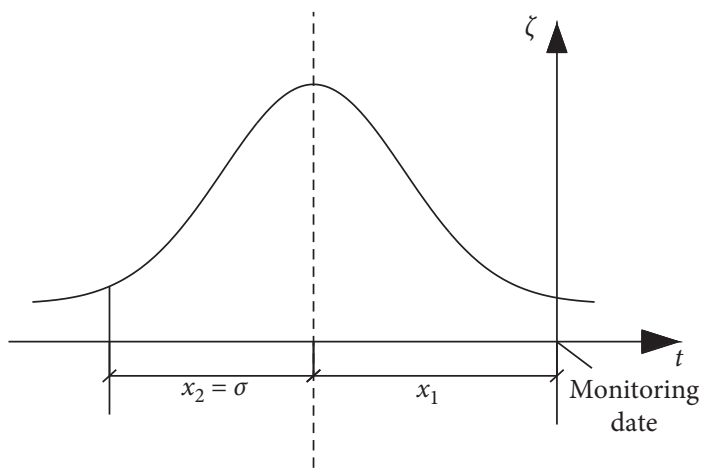

FIgURE 1: The influence of upstream reservoir water level on dam seepage.

reservoir water level at $t$ time. Then, the water level component is

$$
Q_{H}=\sum_{i=1}^{3} a_{i} H_{d}^{i}
$$

2.2.2. Rainfall Component. In order to consider the lag effect of rainfall on seepage of CFRD reasonably, a lag function reflecting the lag effect of rainfall is introduced in this paper [34], which can be expressed as follows:

$$
w(t)=\frac{1}{\sqrt{2 \pi} x_{4} t} e^{-\left(\ln t-x_{3}\right)^{2} / 2 x_{4}^{2}}
$$

where $x_{3}$ is the lagging days and $x_{4}$ is the distribution parameter of rainfall influence.

Suppose the fixed monitoring day $t_{1}$, the starting date of analysis is $t_{0}$; generally, it can be taken as 1 to 2 months before the starting date. There are

$$
\int_{t_{0}}^{t_{1}} w(t) d t=1
$$

Using the exponential transformation method to calculate the rainfall, it can be got that

$$
P_{d}=\int_{t_{0}}^{t_{1}} w(t)[P(t)]^{\beta} d t=\int_{t_{0}}^{t_{1}} \frac{1}{\sqrt{2 \pi} x_{4} t} e^{-\left(\ln t-x_{3}\right)^{2} / 2 x_{4}^{2}}[P(t)]^{\beta} d t,
$$

where $P(t)$ is the rainfall at time $t, P_{d}$ is the equivalent rainfall at time $t_{1}, \beta$ is the infiltration transformation index, $0<\beta<1$, and the other symbols have the same meaning in equation (14).

Thus, the rainfall component can be obtained:

$$
Q_{P}=b P_{d}=b \int_{t_{0}}^{t_{1}} \frac{1}{\sqrt{2 \pi} x_{4} t} e^{-\left(\ln t-x_{3}\right)^{2} / 2 x_{4}^{2}}[P(t)]^{\beta} d t
$$

where $Q_{P}$ is the rainfall component, $x_{3}$ is the lagging days of rainfall, $x_{4}$ is the distribution parameter of rainfall influence, $P_{d}$ is the equivalent rainfall, $b$ is the regression coefficient, and $P(t)$ is the rainfall at $t$ time.
2.2.3. Cracks Component. In the existing CFRDs, there are a lot of cracks in the face slabs of many dams. The influence of cracks on seepage cannot be neglected, but the traditional seepage statistical model does not consider this factor. Based on the principle of slot flow in parallel plates, the equivalent slot width $b_{e i}$ and roughness correction coefficient $C_{i}$ are introduced as cracks component [33-35]. The expression is

$$
Q_{k}=k_{1} \sum_{i=1}^{m} C_{i} b_{e i}^{3} l_{i} H_{i}
$$

where $k_{1}$ is the regression coefficient, $m$ is the number of cracks before the monitoring date, $l_{i}$ is the length of crack, and the roughness correction coefficient $C_{i}$ is calculated according to the following formula: $C_{i}=1 /\left(1+8.8\left(\Delta / 2 b_{e i}\right)^{1.5}\right)$, where $\Delta$ is the absolute roughness of the crack on the face slab.

The seepage safety monitoring model of CFRD with cracks is established by studying the reservoir water level and rainfall component, temperature component, aging component, and cracks component:

$$
\begin{aligned}
Q= & Q_{H}+Q_{P}+Q_{T}+Q_{K} \\
= & a_{0}+\sum_{i=1}^{3} a_{i} H_{d}^{i}+b P_{d}+\sum_{i=1}^{n}\left(c_{1 i} \sin \frac{2 \pi i t}{365}+c_{2 i} \cos \frac{2 \pi i t}{365}\right) \\
& +d_{1} \theta+d_{2} \ln \theta+k_{1} \sum_{i=1}^{m} C_{i} b_{e i}^{3} l_{i} H_{i}
\end{aligned}
$$

where $a_{0}$ is a constant and the other parameters have the same meaning as the above equations.

\subsection{Seepage Safety Monitoring Model of CFRD Based on GA-RBFNN}

2.3.1. Genetic Algorithm (GA). Genetic Algorithms (GAs) originated in the 1960s [36-39]. It is a global parallel, random search optimization algorithm that simulates the inheritance and evolution of organisms in the natural environment and makes the population converge globally. Compared with other optimization algorithms, GA has the following advantages: (1) the encoding of decision variables is the object of operation. By coding decision variables, the evolutionary methods such as genetic and mutation of organisms in a higher degree can be imitated learning from the concepts of biological chromosomes and genes in the calculation process. At the same time, the operation of coding is more convenient. (2) Search information is the objective function to be optimized. Traditional optimization methods need not only objective function but also some other information to help determine the search direction. GA only needs the objective function and finds the fitness function to complete the search task, especially when the objective function cannot be derived. (3) Using the multipoint search method. Single-point search method is often used in conventional optimization algorithms, but its search information is limited and it is easy to fall into local optimum. GA often starts from the most adaptable part of the 
individual. The new generation of population through genetic operation still contains a lot of population information and will not fall into search stagnation. (4) Using probability search technology. Conventional optimization methods use deterministic search, so it is difficult to get the optimal solution. GA uses the probability method to search, and in the process of genetic operation, crossover and mutation are carried out in a certain probability way so that the optimal solution can be easily obtained.

The basic operations are as follows: (1) chromosome coding; (2) fitness function design; (3) selection; (4)crossover and mutation. The steps are as follows:

Step 1: chromosome coding. Because of the need to operate large real numbers, it is more convenient to use real coding. Each parameter to be optimized represents a gene. All parameters to be optimized are combined into a chromosome, and each chromosome corresponds to a complete RBFNN. Single chromosome can be expressed as $C_{11} C_{21} \ldots C_{l 1} C_{12} C_{22} \ldots C_{j k} \ldots C_{l m}$ $\sigma_{1} \sigma_{2} \ldots \sigma_{j} \ldots \sigma_{l} W_{11} W_{21} \ldots W_{n 1} W_{12} \ldots W_{i j} \ldots W_{n l}$, and there are $N$ chromosomes.

Step 2: design of fitness function design. The reciprocal sum of the square error between the output value and the predicted value of the network is taken as the evaluation function of chromosome quality. The fitness values of all individuals are nonnegative. The fitness of a single chromosome is

$$
F=\frac{1}{\sum_{i=1}^{n}\left(f_{i}(X)-y_{i}\right)^{2}},
$$

where $f_{i}$ is the network output value and $y_{i}$ is the target value.

Step 3: selection. Selection is to select good individuals from the old population with a certain probability to form a new population and to reproduce the next generation, that is, to reproduce the operation according to certain conditions. The probability that the first chromosome will be selected is

$$
P_{t}=\frac{F_{t}}{\sum_{1}^{p} F}
$$

where $F_{t}$ is the fitness of the $t^{\text {th }}$ individual. The selection method of roulette is adopted. Firstly, $P_{t}$ of each individual is calculated, and then a number $r \in[0,1]$ is generated randomly. If $P_{1}+P_{2}+\ldots+P_{t-1}<r<P_{1}+P_{2}+\ldots+P_{t}$, then $X_{t}$ is selected. The best preservation strategy can be used to copy the excellent individuals from the population directly to the next generation.

Step 4: cross and mutation. Crossing is the combination of two individuals to produce new genotype individuals. Crossing is the main way to produce new individuals. Because of the optimization of $C_{j k}, \sigma_{j}$, and $W_{i j}$, it is more appropriate to adopt the multipoint crossover method. Two individuals are randomly selected from the population according to the probability, and one crossing point is generated for $C_{j k}, \sigma_{j}$, and $W_{i j}$ according to the crossing probability, and the crossing operation is carried out. The crossover probability is generally $0.65-0.9$. Variation is a secondary means of generating new individuals. The specific operation is to select a pair of individuals from the parent population with a certain probability and then randomly change the three types of gene values with a certain probability. Variant manipulation can effectively prevent useful genes from being lost in genetic manipulation. The probability of variation $P_{m}$ is generally $0.01-0.1$.

\subsubsection{Radial Basis Function Neural Networks (RBFNNs).} RBFNN is a three-layer neural network, which consists of input layer, hidden layer, and output layer [40, 41]. The number of units in each layer is set to $m, l$, and $n$, respectively, and the RBFNN structure is shown in Figure 2. Two neurons in adjacent layers can connect with each other and transmit signals from lower layers to higher layers. Input data are transformed from low-dimensional input layer to high-dimensional hidden layer through a nonlinear function and then linearly mapped from high-dimensional space to output layer. In theory, the network can fit any continuous function, and the accuracy can be arbitrary. The topological structure and parameters of RBFNN have a great influence on the network performance. Generally speaking, $m$ is determined by the number of input variables, $n$ is determined by the number of output variables, and $l$ is determined by the problem setting. The transfer function of hidden layer is called radial basis function. The commonly used transfer functions are Gauss function, multiquadratic function, and inverse multiquadratic function. In this paper, Gauss function is chosen, and equation (22) is as follows:

$$
\phi(t)=\exp \left(-\frac{t^{2}}{\delta_{i}^{2}}\right)
$$

The principle of RBFNN can be described as follows: in the hidden layer, the radial basis function such as Gauss function is used as the basis, and the center of the basis function is expressed as $C_{j}$. The basis function conforms to the centrosymmetric nonlinear distribution. The hidden layer responds to the input mode after $C_{j}$ is determined according to the basis function. If the weight $W_{i j}$ from the hidden layer to the output layer is obtained, the whole network structure is determined. The principle can be expressed in Figure 3.

$\mathrm{P}$ samples are selected, given input mode $X_{k}=\left(x_{1}, x_{2}, \ldots x_{m}\right)$, and then the output of hidden layer unit is obtained by the following equation:

$$
\phi_{j}(X)=\exp \left[-\frac{1}{2} \sum_{k=1}^{m}\left(\frac{x_{k}-C_{j k}}{\sigma_{j}}\right)^{2}\right],
$$

where $\phi(\cdot)$ is the Gauss function, $C_{j}=\left(C_{j 1}, C_{j 2}, \ldots\right.$, $\left.C_{j k}, \ldots, C_{j m}\right)$ is the central vector of the $j^{\text {th }}$ hidden layer node, whose dimension is equal to the number of input variables, and $\sigma_{j}$ is the central width of the $j^{\text {th }}$ hidden layer node. 


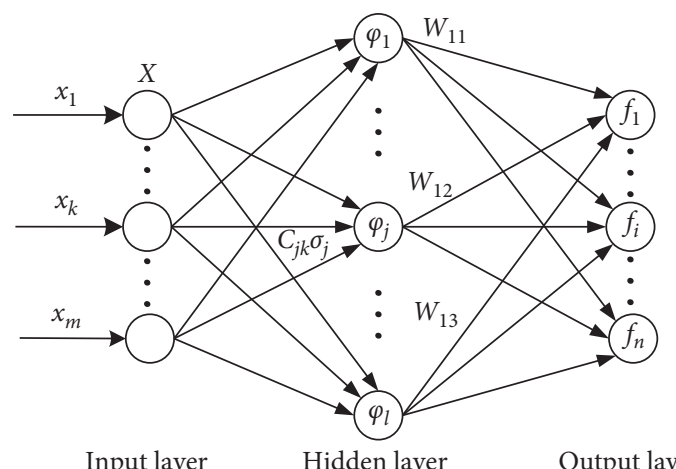

FIGURE 2: Structure of RBFNN.

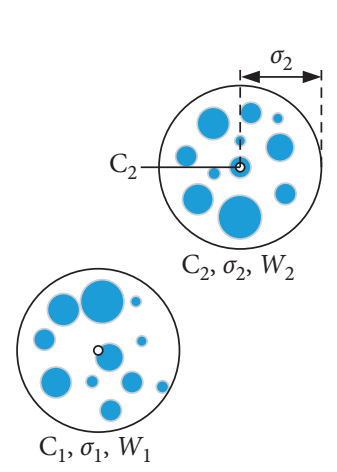

Figure 3: Principle of RBFNN.

Output values are got from the hidden layer through the following equation:

$$
f_{i}(X)=\sum_{j=1}^{n} W_{i j} \phi_{j}(X),
$$

where $f_{i}(X)$ is the output value of the $i^{\text {th }}$ output layer and $W_{i j}$ is the connection weight.

In this study, in order to get better parameters of RBFNN, GA is used to optimize $C_{j k}, \sigma_{j}$, and $W_{i j}$.

2.3.3. GA Optimization of RBFNN. In order to better simulate the service behavior of CFRD with RBFNN, GA is used to optimize the parameters of RBFNN. The basic idea is to train $C_{j k}, \sigma_{j}$, and $W_{i j}$ of RBFNN with GA, to find a better solution, and then train the RBFNN with the result as the initial parameter. The main steps are as follows:

Step 1: data normalization. The original data are normalized to facilitate subsequent operations, and the appropriate data are selected as training samples.

Step 2: chromosome coding. Using real number coding, a single chromosome contains $m+n+l$ genes and generates the initial population $P(g)$. The evolutionary algebra is denoted as $g=0$, and the maximum evolutionary algebra $G$ is determined.

Step 3: chromosome decoding. $C_{j k}, \sigma_{j}$, and $W_{i j}$ are got, and the output layer value $f_{i}(X)$ is calculated by equation (24).
Step 4: calculate fitness. The fitness value $F$ of each individual is calculated by equation (20) to evaluate the performance of the network. If $g>G$, it will end; otherwise, go to next step.

Step 5: $g=g+1$, and do genetic manipulation.

(a) According to the fitness $F$ of individuals, individuals are selected to enter the next generation population $P(g+1)$ by roulette gambling. Individuals with high fitness can be directly copied to their offspring and paired with other chromosomes.

(b) Cross-manipulation of paternal chromosomes with probability $P_{c}$ to obtain new chromosomes.

(c) Three genes of selected chromosomes were mutated by probability $P_{m}$ to obtain new chromosomes.

Step 6: generate a new population through Step 5 and transfer to Step 4 until D.

The specific algorithm flow is shown in Figure 4.

2.4. Calculation of Component Proportion. According to the monitoring data of seepage, the GA-RBFNN model is established by using monitoring data and environmental factors. The input variables of model are water level, temperature, aging, cracks, and so on. Seepage is the output of the network. Assuming that the number of nodes in the input layer is $N$ and the number of nodes in the hidden layer is $M$, considering the specific problems in this paper, the number of nodes in the output layer is 1 . Let $C_{i j}$ and $\sigma_{i j}$ be the connection weight between the input layer and the hidden layer and $W_{i 1}$ the connection weight between the hidden layer and the output layer. Steps are as follows: (1) initialization of output node determination, the number of output nodes is $K_{0}$, and it is generally taken as $Q_{0}=1 / K_{0}$; in this paper, $Q_{0}=1$. (2) Inversely find the determinacy of hidden layer node; that is, the determinacy of output layer node is propagated forward by weight function because the determinacy of output node is 1 , so the determinacy of hidden layer node is $1 \times W_{i 1}=W_{i 1}$. (3) Find the degree of certainty of the input layer. For each node $i$ of the hidden layer, each node $j$ of the input layer and then $p_{i j}$ can be obtained:

$$
p_{i j}=\frac{\left|x_{j}-C_{i j} / \sigma_{i}\right|}{\sum_{j=1}^{m}\left|x_{j}-C_{i j} / \sigma_{i}\right|} \cdot W_{i 1} .
$$

Standardize $p_{i j}$ and $q_{i j}=p_{i j} /\left(\sum_{j=1}^{N} p_{i j}\right)$. For each input node $j$, sum $q_{i j}$ to get the certainty of input layer and $S_{j}=\sum_{i=1}^{M} q_{i j}$.

Then, the proportion of the influence of each input variable on the output variable (seepage) is as follows:

$$
\mathrm{RI}_{j}=\frac{S_{j}}{\sum_{j=1}^{N} S_{j}} \times 100 \% .
$$

2.5. Calculation of Monitoring Indexes. Firstly, the noise is eliminated by wavelet on the safety monitoring data, and then the digital feature Ex, En, and $\mathrm{He}$ of the sample is 


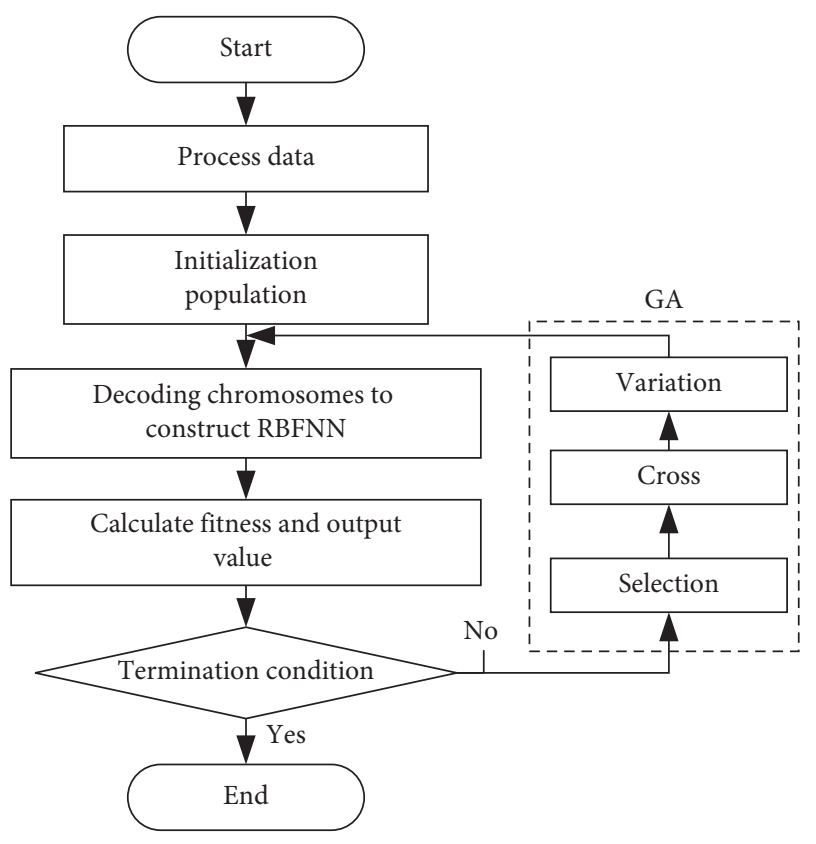

FIgURE 4: Algorithm flow of GA-RBFNN.

obtained by the reverse cloud generator; then, $n$ cloud droplets are generated by the forward cloud generator, and the corresponding determinacy $\mu_{i}$ is calculated according to the following equation:

$$
\mu_{i}=\exp \frac{-\left(x_{i}-E x\right)^{2}}{2 E n_{i}^{2^{\prime}}} .
$$

Through the above operation, the monitoring effect of unknown distribution of noise is transformed into known probability density and determinacy cloud droplet group. The contribution of $\Delta x$ to $C$ can be obtained as follows:

$$
\Delta C=\frac{\mu_{A}(x) \Delta x}{\sqrt{2 \pi} E n} .
$$

According to the " $3 E n$ rule" of the forward cloud, it can be found that the cloud droplets on the interval $[E x-3 E n, E x+3 E n]$ have a major contribution to $C$. In addition, the contribution of cloud droplets on the interval $(-\infty, E x-3 E n)$ and $(E x+3 E n,+\infty)$ to $C$ is very small, and the probability of occurrence is very small, so it can be regarded as abnormal monitoring information:

$$
\frac{1}{\sqrt{2 \pi} E n} \int_{E x-3 E n}^{E x+3 E n} \mu_{T}(x) d x=99.74 \% .
$$

\section{Case Study}

3.1. Engineering Introduction. The highest body of one CFRD is $120.0 \mathrm{~m}$, and the elevation of its top is $760.00 \mathrm{~m}$; the dam crest extends as long as $259.8 \mathrm{~m}$ from east to west, and the face is as thick as $t=0.3+0.00347 H(\mathrm{~m})$, which changes linearly from up to down, that is, $0.3 \mathrm{~m}$ at the top and $0.7 \mathrm{~m}$ at the bottom. The upstream slope is $1: 1.4$, whereas the downstream slope is $1: 1.35$. On February 6, 2004, the faces were checked and 180 cracks were found. In 2012, a total of 206 cracks were found, and the distribution of face cracks is shown in Figure 5.

On the basis of the landform and structure, the seepage discharge at the two measuring weirs, namely, W1 and W2, is mainly the abutment seepage. The retaining wall set where the plant meets the dam foot leads the seepage to a certain location, where W3 was set. The seepage discharge at W3 is face, base, and W1 and W2 seepages. The distribution of measuring weirs is shown in Figure 6. The seepage discharge at $\mathrm{W} 1$ and $\mathrm{W} 2$ is relatively small, indicating that the antiseepage at the two banks is effective.

3.2. Determination of Each Component. The hydraulic component $\delta_{H}$ is expressed by the water depth in front of the dam: $H_{d}, H_{d}^{2}$, and $H_{d}^{3}$. Temperature component $\delta_{T}$ is expressed by multiple harmonic combinations: $\sin 2 \pi i t / 365-$ $\sin 2 \pi i t_{0} / 365$ and $\cos 2 \pi i t / 365-\cos 2 \pi i t_{0} / 365$. The selected data are annual periodic, so $i=1$, and $t$ is the cumulative days from the start of the series to the monitoring day. Factors of rainfall component $\delta_{P}$ is $P_{d}$. The aging component $\delta_{\theta}$ is expressed by polynomials and logarithmic functions: $\theta-\theta_{0}$, $\ln \theta-\ln \theta_{0}$, where $\theta=t / 100, \theta_{0}=t_{0} / 100$, and $t$ is the cumulative day from the initial monitoring day to the monitoring day.

As for the cracks component, because it takes a huge amount of manpower, material, and financial resources to implement a complete crack detection, there are only two times of crack detection, which belongs to short time series monitoring data. In view of the characteristics of small sample and poor information such as face cracks, this paper regards the dam as a grey system and regards the short monitoring data of measured cracks as a discrete random sequence. From 2000 to 2004, most of the cracks were caused by construction and concrete quality, which had great uncertainty and randomness. Therefore, this paper assumes that 180 cracks were randomly generated during the first inspection. From 2004 to 2012, the number of cracks increased by 26 and tended to converge. At this time, temperature component is the main cause for cracks occurring in the upper part of face slab. Most cracks occur at low temperature, and the expression of cracks component is $\sum_{i=1}^{m} C_{i} b_{e i}^{3} l_{i} H_{i}$.

\subsection{Seepage Safety Monitoring Model Based on GA-RBFNN.} The historical monitoring upstream water level and rainfall are shown in Figure 7, and the monitoring seepage after noise reduction is shown in Figure 8. Table 1 only lists 26 cracks on the largest fracture surface relative to the distribution height $z_{i}$, equivalent width $b_{e i}$, and relative roughness $\Delta / 2 b_{e i}$ of the dam base.

9 factors of water pressure, temperature, rainfall, aging, and cracks are selected as input variables and seepage as output variables. RBFNN with input layer 9, output layer 1, and hidden layer 10 is constructed. GA is used to optimize $C_{j k}, \sigma_{j}$, and $W_{i j}$, and the optimized $C_{j k}, \sigma_{j}$, and $W_{i j}$ is used as the initial value of RBFNN for training and prediction. From December 11, 2000, to August 15, 2013, there were 600 


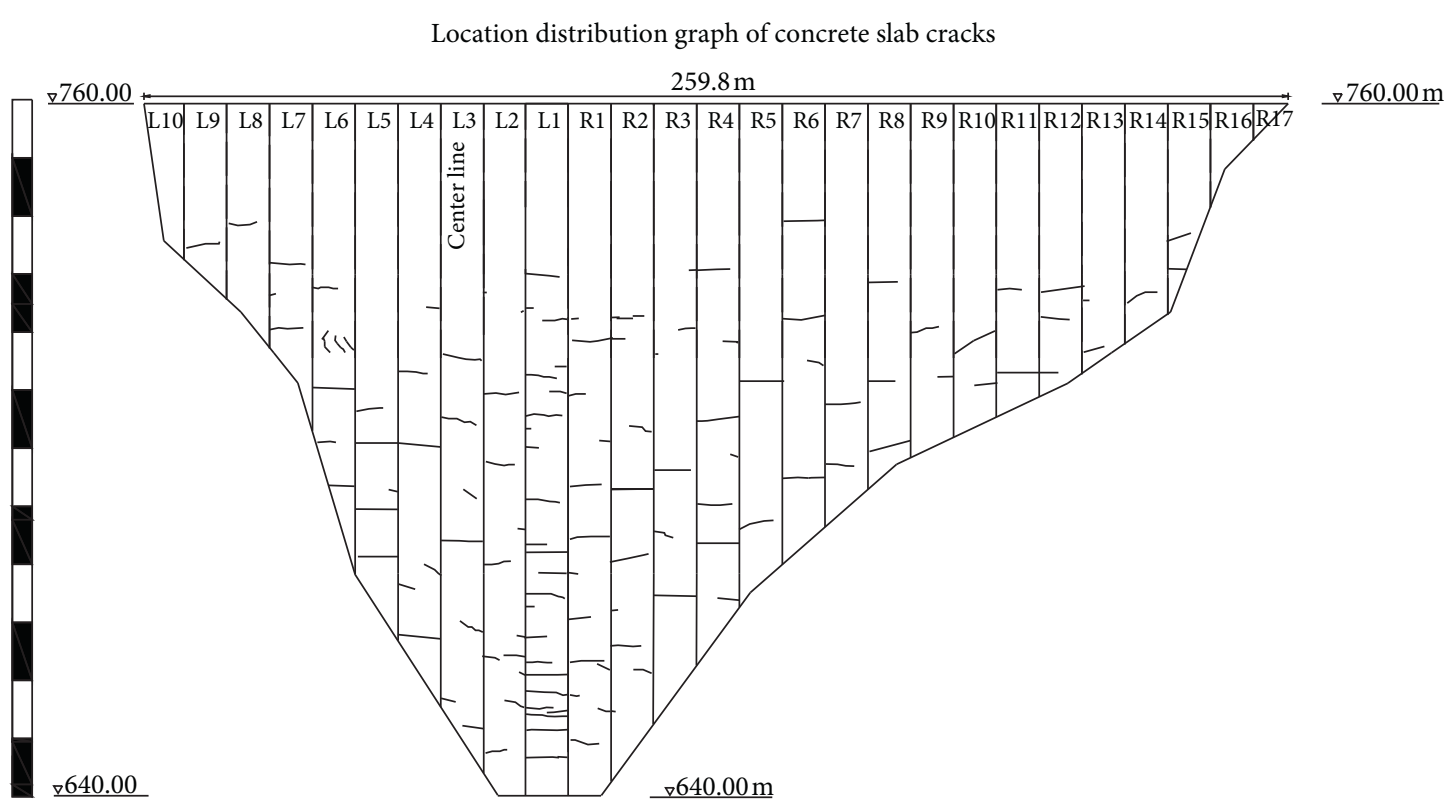

FIGURE 5: Distribution of face cracks.

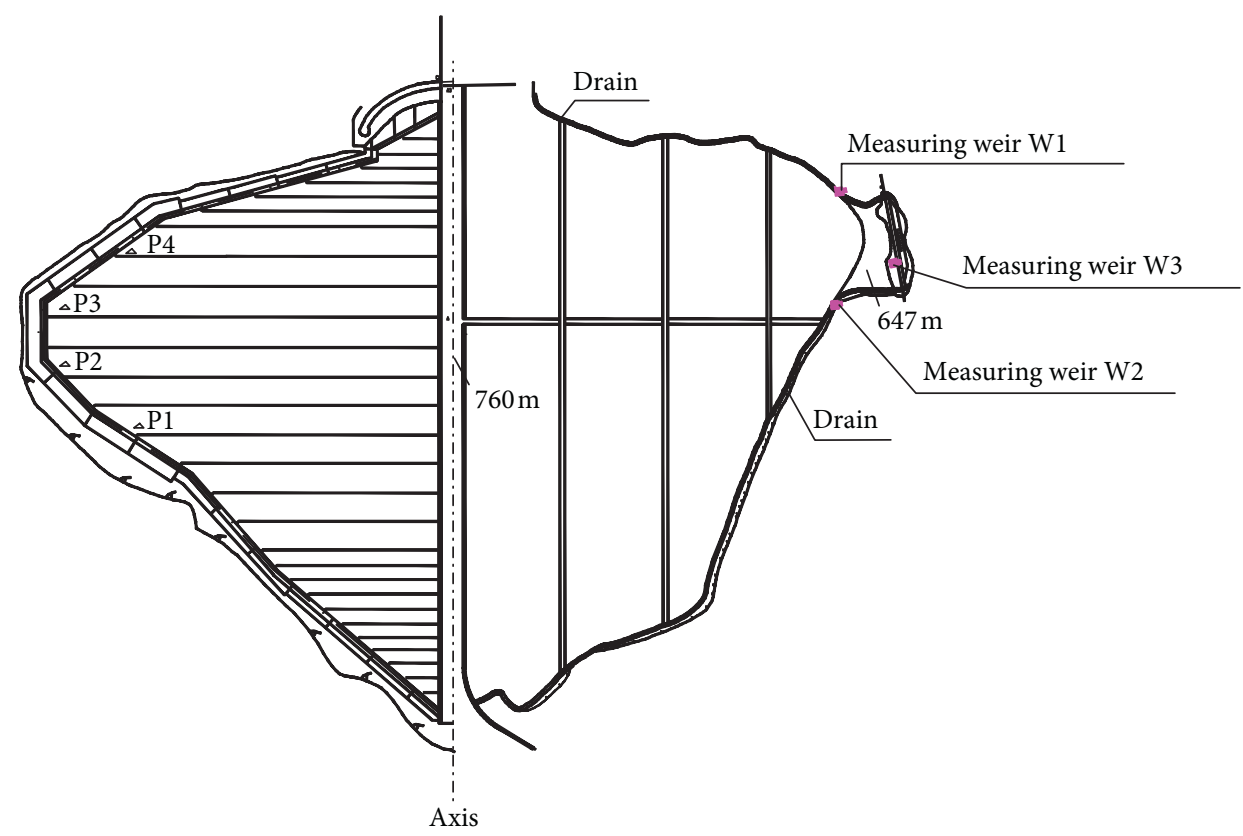

FIgURE 6: Distribution of measuring weirs.

groups of monitoring data in the seepage safety monitoring model. 450 groups of data were selected as training samples, and 150 groups of data were selected as test samples. Through continuous experiments and comprehensive consideration, the parameters of GA are set in Table 2. The standard RBFNN model and GA-RBFNN model are used for training and prediction, respectively. The results are shown in Figure 9, and the error curve is shown in Figure 10.

By comparing the mean square error (MSE) and correlation coefficient $(\mathrm{R})$ of the two models, the advantages and disadvantages of the two models are determined. The mean square error is calculated according to the following equation:

$$
\mathrm{MSE}=\frac{1}{n} \sum_{1}^{n}(f-y)^{2},
$$

where $f$ is the output value and $y$ is the monitoring data. The mean square error (MSE) and correlation coefficient (R) are shown in Table 3.

From Figures 9 and 10, it can be seen that the GARBFNN model is better than the standard RBFNN model in 


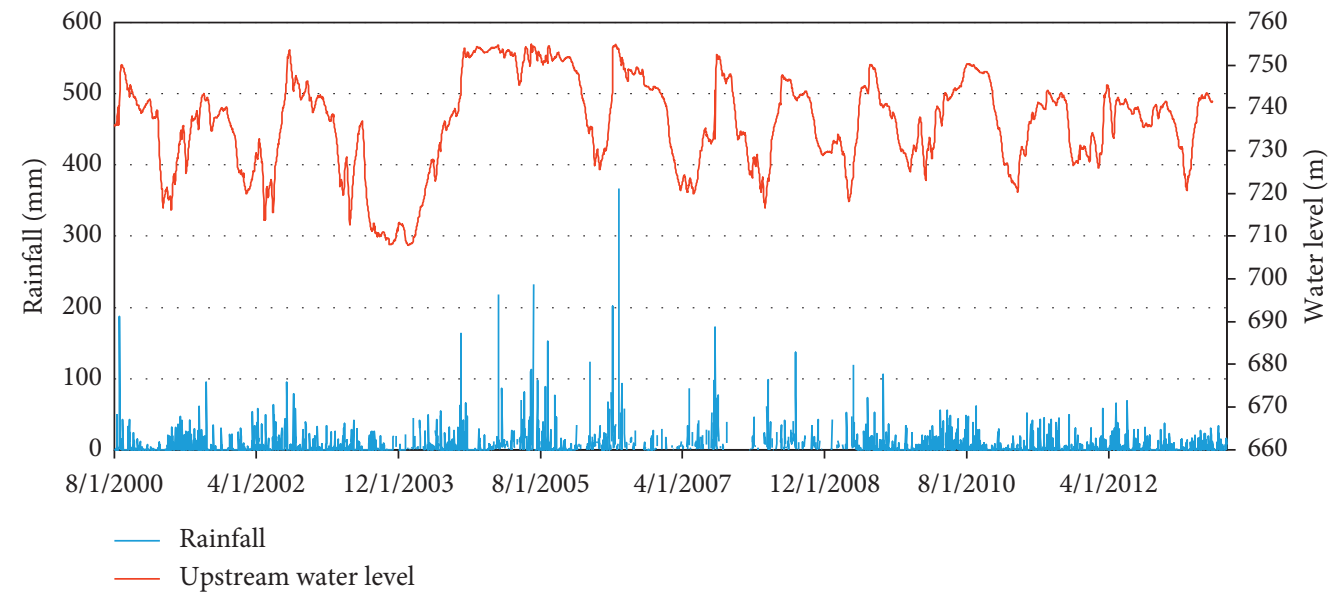

Figure 7: The historical monitoring upstream water level and rainfall.

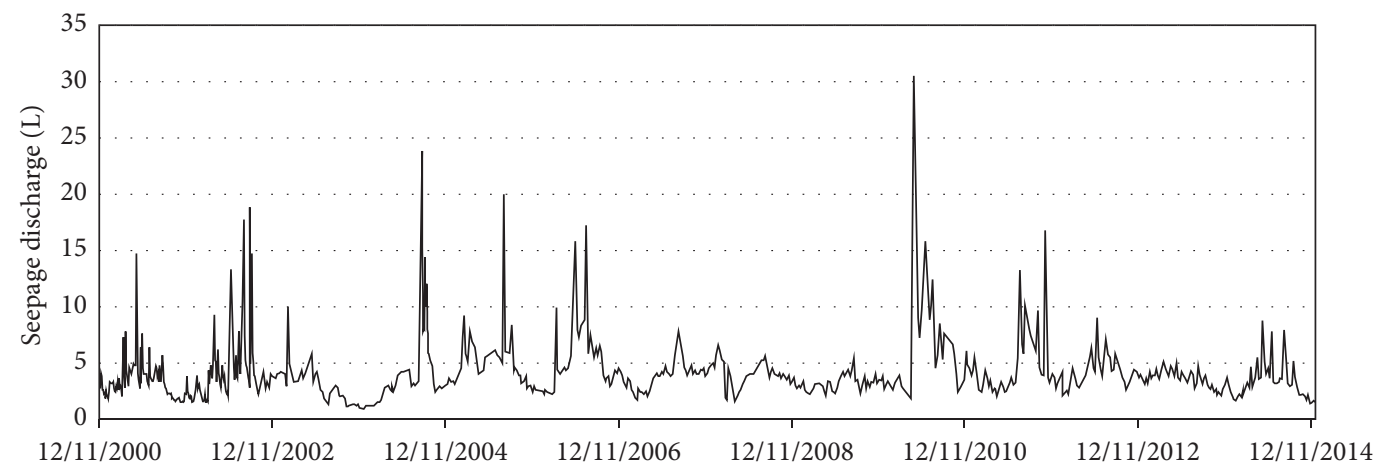

FIGURE 8: The monitoring seepage after noise reduction.

TABLE 1: Parameters of cracks.

\begin{tabular}{lccccccccc}
\hline$i$ & $z_{i}(m)$ & $b_{e i}(m m)$ & $l_{i}$ & $\Delta / 2 b_{e i}$ & $i$ & $z_{i}(m)$ & $b_{e i}(m m)$ & $\Delta / 2 b_{e i}$ & $l_{i}$ \\
\hline 1 & 7.98 & 0.05 & 3.53 & 0.30 & 14 & 48.89 & 0.31 & 0.31 & 10.33 \\
2 & 8.51 & 0.22 & 7.98 & 0.34 & 15 & 53.01 & 0.37 & 0.24 \\
3 & 12.89 & 0.17 & 6.11 & 0.29 & 16 & 56.64 & 0.41 & 0.43 \\
4 & 13.15 & 0.16 & 6.23 & 0.34 & 17 & 56.81 & 0.29 & 0.31 \\
5 & 14.47 & 0.12 & 6.68 & 0.21 & 18 & 77.42 & 0.28 & 0.34 \\
6 & 17.96 & 0.24 & 3.49 & 0.27 & 19 & 77.53 & 0.07 & 0.36 \\
7 & 26.39 & 0.33 & 2.94 & 0.23 & 20 & 86.77 & 0.40 & 0.29 \\
8 & 29.90 & 0.37 & 7.73 & 0.27 & 21 & 86.96 & 0.35 & 0.03 \\
9 & 30.62 & 0.48 & 9.92 & 0.24 & 22 & 87.42 & 0.28 & 0.31 \\
10 & 34.21 & 0.23 & 7.60 & 0.46 & 23 & 90.34 & 0.38 & 5.28 \\
11 & 34.60 & 0.46 & 6.64 & 0.33 & 24 & 90.85 & 0.26 & 0.26 \\
12 & 40.55 & 0.43 & 5.44 & 0.21 & 25 & 95.11 & 0.16 & 0.29 & 0.49 \\
13 & 43.93 & 0.05 & 8.37 & 0.30 & 26 & 106.75 & 0.13 & 3.92 \\
\hline
\end{tabular}

fitting and forecast dam seepage, and the GA-RBFNN model is closer to the monitoring data than the standard RBFNN model. Table 3 shows that, in the fitting part, the MSE of the GA-RBFNN model is better than that of the RBFNN model, as well as in the prediction part; in addition, the R of GARBFNN model is also better than that of the RBFNN model. Based on the analysis of MSE and R, it can be shown that the fitting and prediction accuracy of RBFNN can be improved by using GA. The model can be applied to the seepage monitoring of long-term service of dams.
3.4. Calculation of Component Proportion. The proportion of each component can be obtained by summing the proportions of the factors included in the water pressure component, the temperature component, rainfall component, the aging component, and cracks component, respectively. The results are shown in Table 4 .

It can be seen from Table 4 that the monitoring data of seepage are mainly affected by rainfall, about 50\%; the water pressure component is the second factor, about $20 \%$, and the effect of aging component is smaller, which is 
TABLe 2: The parameters of GA.

\begin{tabular}{lccccc}
\hline Total samples & Population size $p$ & Samples for forecast & Crossover probability $P_{c}$ & Mutation probability $P_{m}$ & Training generations $G$ \\
\hline 600 & 450 & 150 & 0.70 & 0.05 & 1000
\end{tabular}

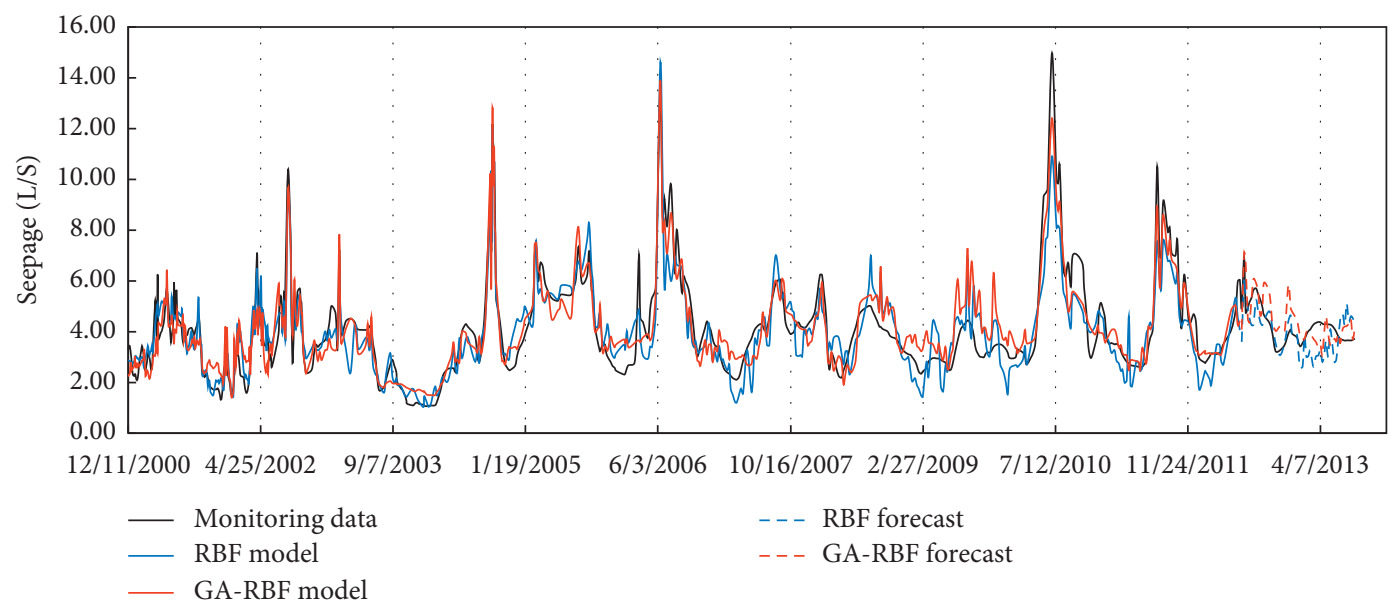

FIGURE 9: Comparison of fitting value and prediction value with monitoring data (RBFNN and GA-RBFNN) in WE-3 (L/s).

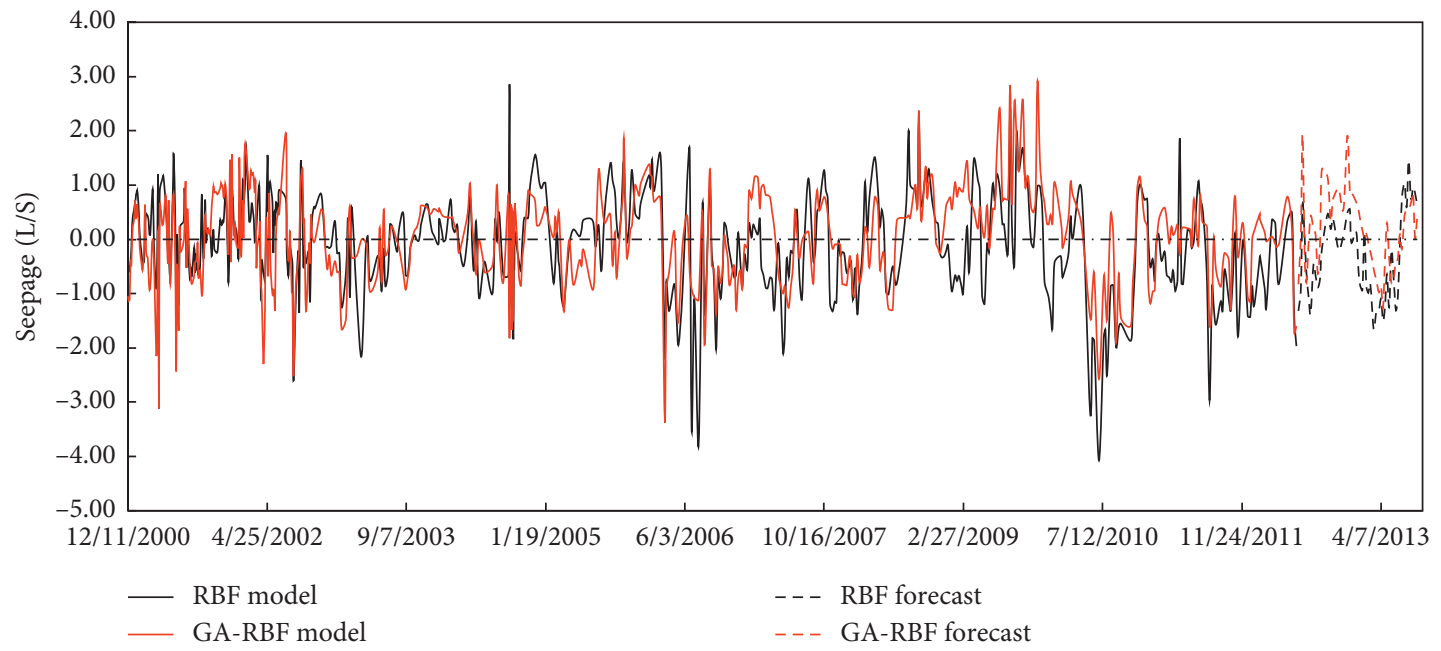

FIGURE 10: Error of fitting value and forecast value with monitoring data (RBFNN and GA-RBFNN) in WE-3 (L/s).

TABLE 3: MSE and R of two models.

\begin{tabular}{lcccc}
\hline Model & Forecast $R$ & MSE & Forecast & Total samples \\
\hline RBFNN & & Fitting & 0.28 & 0.78 \\
GA-RBFNN & 0.924 & 1.23 & 0.17 & 0.67 \\
\hline
\end{tabular}

TABLE 4: Component proportion.

\begin{tabular}{lccccc}
\hline Model & Water pressure (\%) & Temperature (\%) & Rainfall (\%) & Aging (\%) & Cracks (\%) \\
\hline Stepwise regression & 23 & 10 & 54 & 8 & 5 \\
RBFNN & 21 & 11 & 55 & 5 & 7 \\
GA-RBFNN & 19 & 12 & 52 & 10 \\
\hline
\end{tabular}

basically consistent with the conclusion of the statistical model. In addition, the influence of the crack component introduced in this paper is about $10 \%$, which cannot be ignored. It shows that the introduction of crack component has a certain practical significance for safety monitoring. 


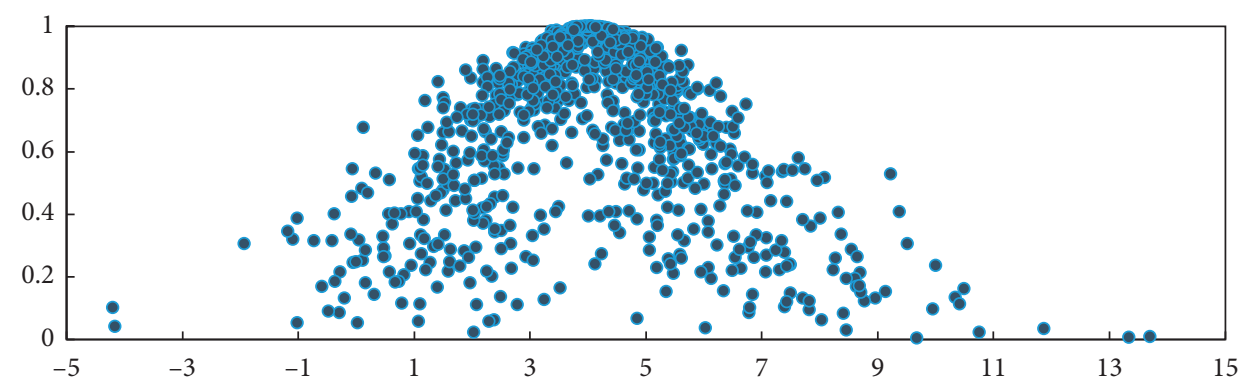

FIgURE 11: The cloud images of CFRD seepage $(E x=4.0194, E n=1.6926$, and $\mathrm{He}=0.8917)$.

3.5. Calculation of Monitoring Indexes. From December 11, 2000, to August 15, 2013, a total of 600 groups of seepage monitoring data were processed by wavelet denoising. Firstly, the inverse cloud generator is used to get the stereotype concept $C(E x, E n$, and $\mathrm{He})$ of denoising data, and then, 600 cloud droplets satisfying the requirements are generated according to Ex, En, and $\mathrm{He}$ forward cloud models. According to the " $3 E n$ rule," the safe interval values of CFRD's seepage are obtained. The cloud images are shown in Figure 11. The results are as follows:

$$
\begin{aligned}
(E x, E n, H e) & =(4.0194,1.6926,0.8917), \\
{[E x-3 E n, E x+3 E n] } & =[-1.0584,9.0971] .
\end{aligned}
$$

It can be seen from Figure 11 that when the monitoring data of seepage are greater than $9.0971 \mathrm{~L} / \mathrm{s}$, component analysis is needed to exclude the possibility of abrupt change in measured value caused by structural variation.

\section{Conclusions}

Aiming at the problem that the traditional regression model does not have good applicability to simulate the influence of water level and rainfall in the early stage based on experience, the normal distribution curve is used to simulate the lagging effect of water level and rainfall on seepage in this paper. Considering the influence of face cracks on seepage, the face crack is regarded as an influencing factor. A seepage safety monitoring model of CFRD with cracks considering the lagging effect is proposed. The seepage monitoring model is optimized by using GA-RBFNN, and the seepage is predicted by using this model. The prediction results are not much different from the monitoring data, and the prediction results obtained by the GA-RBFNN model are better than those obtained by the traditional RBFNN model, which shows that the prediction results proposed in this paper are better than those obtained by the traditional method. The method has certain applicability. Through the analysis of the component proportion of each factor, it can be seen that the rainfall component has the greatest influence on the total seepage, accounting for $52 \%$, which may be caused by the distance between the weir and the dam site, and the rainfall flowing into the weir through the mountain body; the crack component accounts for about $10 \%$ of the total seepage, so the influence of the crack component on the total seepage flow cannot be ignored. Finally, through the cloud model, the seepage monitoring index of the CFRD is worked out, which has certain guiding significance for the treatment of abnormal seepage monitoring data.

The seepage safety monitoring model of CFRD with slab cracks is based on monitoring data, and using the finite element method to simulate and calculate the proportion of each component is the future work. Analyzing the differences between the proposed model in this paper and FEM model will be an interesting work.

\section{Data Availability}

The data used to support the findings of this study are available from the corresponding author upon request.

\section{Conflicts of Interest}

The authors declare that there are no conflicts of interest.

\section{Authors' Contributions}

Zhongwen Shi and Bo Xu contributed equally to this work.

\section{Acknowledgments}

This study was funded by the National Natural Science Foundation of China (Grant nos. 51739003, 51479054, 51779086, 51579086, 51379068, 51579083, 51579085, 51609074, and 51609149), National Key R\&D Program of China (2016YFC0401601, 2017YFC0804607, and 2018YFC0407104), Priority Academic Program Development of Jiangsu Higher Education Institutions (YS11001), Jiangsu Natural Science Foundation (Grant no. BK20160872), Special Project Funded of National Key Laboratory (20165042112), Key R\&D Program of Guangxi (AB17195074), and Central University Basic Research Project (2017B11114 and 2018B25514).

\section{References}

[1] D. Zou, H. Han, J. Liu, D. Yang, and X. Kong, "Seismic failure analysis for a high concrete face rockfill dam subjected to near-fault pulse-like ground motions," Soil Dynamics and Earthquake Engineering, vol. 98, pp. 235-243, 2017.

[2] Y. Q. Shi, J. J. Yang, J. L. Wu et al., "A statistical model of deformation during the construction of a concrete face rockfill dam," Structural Control and Health Monitoring, vol. 25, Article ID e2074, 2018. 
[3] B. Zhang, R. Wang, and R. F. Shi, "Time-dependent deformation in high concrete-faced rockfill dam and separation between concrete face slab and cushion layer," Computers and Geotechnics, vol. 31, no. 7, pp. 559-573, 2004.

[4] X. Q. Niu, "Safety and consideration of high face rockfill dams," Journal of Hydropower, vol. 36, pp. 104-111, 2017.

[5] N. H. Yun and Z. Y. Yang, "Technical progress of China concrete face rockfill dam," Journal of Geotechnical Engineering, vol. 34, pp. 1361-1368, 2012.

[6] S. Khalid, B. Singh, G. C. Nayak, and O. P. Jain, "Nonlinear analysis of concrete face rockfill dam," Journal of Geotechnical Engineering, vol. 116, no. 5, pp. 822-837, 1990.

[7] R. Wang, Q. Sun, D. Ma, and Z. Liu, "The small-signal stability analysis of the droop-controlled converter in electromagnetic timescale," IEEE Transactions on Sustainable Energy, vol. 10, no. 3, pp. 1459-1469, 2019.

[8] Y. Xiang, S.-y. Fu, K. Zhu, H. Yuan, and Z.-y. Fang, "Seepage safety monitoring model for an earth rock dam under influence of high-impact typhoons based on particle swarm optimization algorithm," Water Science and Engineering, vol. 10, no. 1, pp. 70-77, 2017.

[9] C. Xiang, L. Qingquan, Z. Zhiwei et al., "Research on a seepage monitoring model of a high core rockfill dam based on machine learning," Sensors, vol. 18, no. 9, p. 2749, 2018.

[10] C. S. Gu and Z. R. Wu, Theory and Method of Dam and Dam Foundation Safety Monitoring and its Application, Hohai University Press, Nanjing, China, 2006.

[11] C. S. Shen and Z. R. Wu, "Discussion on statistical model of uplift pressure of concrete dam foundation," Journal of Hehai University: Natural Science Edition, pp. 109-116, 1989.

[12] D. R. Cen, Study on Seepage Field Characteristics and Analytical Model of Overburden Rockfill Dam Area, Hohai University, Nanjing, China, 2007.

[13] Q. F. Zhang, C. S. Gu, and Z. R. Wu, "Seepage monitoring model of earth-rock dam based on lag effect," Journal of Water Resources, vol. 1, pp. 85-89, 2001.

[14] T. T. Cai, H. Z. Su, C. S. Gu et al., "Statistical model of dam seepage considering the effect of seepage lag and the change rate of reservoir water level," Hydraulic and Hydropower Technology, vol. 44, p. 45, 2013.

[15] Y. Chen, R. Hu, W. Lu, D. Li, and C. Zhou, "Modeling coupled processes of non-steady seepage flow and non-linear deformation for a concrete-faced rockfill dam," Computers \& Structures, vol. 89, no. 13-14, pp. 1333-1351, 2011.

[16] Z. K. Xu, S. J. Peng, B. W. Wei et al., "Seepage monitoring model of deep overburden upper slab rockfill dam based on cloud adaptive genetic algorithm," Hydropower Energy Science, vol. 31, no. 12, pp. 58-61, 2014.

[17] B. Chen, C. Gu, T. Bao, B. Wu, and H. Su, "Failure analysis method of concrete arch dam based on elastic strain energy criterion," Engineering Failure Analysis, vol. 60, pp. 363-373, 2016.

[18] F. Hooshyaripor, S. Tahershamsi, and S. Golian, "Application of copula method and neural networks for predicting peak outflow from breached embankments," Journal of HydroEnvironment Research, vol. 8, no. 3, pp. 292-303, 2014.

[19] J. Mata, J. Tavares de Castro, and J. S. D. Sá da Costa, "Constructing statistical models for arch dam deformation," Structural Control and Health Monitoring, vol. 21, no. 3, pp. 423-437, 2014.

[20] B. Stojanovic, M. Milivojevic, N. Milivojevic, and D. Antonijevic, "A self-tuning system for dam behavior modeling based on evolving artificial neural networks," Advances in Engineering Software, vol. 97, pp. 85-95, 2016.
[21] D. Venkatesan, R. Kannan, and R. Saravanan, "A genetic algorithm-based artificial neural network model for the optimization of machining processes," Neural Computing and Applications, vol. 18, no. 2, pp. 135-140, 2009.

[22] J. Mata, "Interpretation of concrete dam behaviour with artificial neural network and multiple linear regression models," Engineering Structures, vol. 33, no. 3, pp. 903-910, 2011.

[23] Z. S. Tian, X. N. Zhang, Q. L. Zhu et al., "Study of Bp neural network model to dam deformation monitoring," in Proceedings of the 2010 Sixth International Conference on Natural Computation, Yantai, China, August 2010.

[24] K. Zhang, Safety Monitoring Model and Fuzzy Evaluation of Safety Behavior of Earth-Rock Dams Based on Support Vector Machine, Xi'an University of Technology, Xi'an, China, 2017.

[25] B. Stojanovic, M. Milivojevic, M. Ivanovic, N. Milivojevic, and D. Divac, "Adaptive system for dam behavior modeling based on linear regression and genetic algorithms," Advances in Engineering Software, vol. 65, pp. 182-190, 2013.

[26] X. H. Wu and Z. R. Wu, "2u-Drafting method of deformation monitoring index for roller compacted concrete dam," Water Conservancy and Hydropower Technology, vol. 35, pp. 136138, 2004.

[27] H. Q. Guo, X. H. Wu, Z. R. Wu et al., "Draw up deformation monitoring index of RCC dam by two coupled viscoelastic finite element method," in Proceedings of the National Conference on Structural Engineering, pp. 852-856, Nanjing, China, 2001.

[28] P. J. Cong, C. S. Gu, and Y. C. Gu, "Maximum entropy method for dam safety monitoring indicators," Journal of Wuhan University: Information Science Edition, vol. 33, pp. 1126-1129, 2008.

[29] K. Zhu, D. Qin, L. Wang et al., "Application of cloud model in drafting dam safety monitoring indicators," Hydropower Energy Science, vol. 31, no. 3, pp. 65-68, 2013.

[30] H. Su, Z. Chen, and Z. P. Wen, "Performance improvement method of support vector machine-based model monitoring dam safety," Structural Control and Health Monitoring, vol. 23, no. 2, pp. 252-266, 2016.

[31] D. X. Hu, Z. Q. Zhou, Y. Li, and X. L. Wu, "Dam safety analysis based on stepwise regression model," Advanced Materials Research, vol. 204-210, pp. 2158-2161, 2011.

[32] W. Shaowei, X. Yingli, G. Chongshi et al., "Monitoring models for base flow effect and daily variation of dam seepage elements considering time lag effect," Water Science and Engineering, vol. 11, no. 4, pp. 344-354, 2018.

[33] J. Hu, F. Ma, and S. Wu, "Comprehensive investigation of leakage problems for concrete gravity dams with penetrating cracks based on detection and monitoring data: a case study," Structural Control and Health Monitoring, vol. 25, no. 4, Article ID e2127, 2017.

[34] Q. Jianchun, Z. Dongjian, and Z. Kai, "Seepage monitoring models study of earth-rock dams influenced by rainstorms," Mathematical Problems in Engineering, vol. 2016, Article ID 1656738, 11 pages, 2016.

[35] Y. Li, "Seepage calculation model for face slab joint and crack of concrete face rockfill dam," Chinese Journal of Applied Mechanics, vol. 27, pp. 145-150, 2010.

[36] K. Deb, A. Pratap, S. Agarwal, and T. Meyarivan, "A fast and elitist multiobjective genetic algorithm: NSGA-II," IEEE Transactions on Evolutionary Computation, vol. 6, no. 2, pp. 182-197, 2002.

[37] U. Maulik and S. Bandyopadhyay, "Genetic algorithm-based clustering technique," Pattern Recognition, vol. 33, no. 9, pp. 1455-1465, 2000. 
[38] G. Jones, P. Willett, R. C. Glen, A. R. Leach, and R. Taylor, "Development and validation of a genetic algorithm for flexible docking 1 1Edited by F. E. Cohen," Journal of Molecular Biology, vol. 267, no. 3, pp. 727-748, 1997.

[39] C. Xu, C. Yue, and C. Deng, "Hybrid GA/SIMPLS as alternative regression model in dam deformation analysis," Engineering Applications of Artificial Intelligence, vol. 25, no. 3, pp. 468-475, 2012.

[40] G. Manthei and K. Plenkers, "Review on in situ acoustic emission monitoring in the context of structural health monitoring in mines," Applied Sciences, vol. 8, no. 9, 2018.

[41] M.-J. Chang, H.-K. Chang, Y.-C. Chen et al., "A support vector machine forecasting model for typhoon flood inundation mapping and early flood warning systems," Water, vol. 10, no. 12, p. 1734, 2018. 